connections, or synapses, also seem to be less able to strengthen with repeated activation - changes that could help to explain declines in learning and memory over time. Christine Gall, Gary Lynch and their colleagues at the University of California, Irvine, preserved neuronal branching and boosted synapse strengthening in rats by treating them with a molecule that enhanced the activity of the AMPA-type glutamate receptor. This increased excitatory brain signalling. Animals treated for three months also showed improvements in some tests of long-term memory.

The authors suggest that the mechanism could one day be used to protect against agerelated neuronal decline. J. Neurosci. 36, 1636-1646 (2016)

\title{
PALAEONTOLOGY \\ Mammal with a dinosaur nose
}

A wildebeest-like creature that lived in Africa 70,000 years ago had a trumpet-shaped nasal passage, similar in shape to those of some dinosaurs.

Rusingoryx atopocranion was previously known from incomplete specimens, but a team led by Haley O'Brien at Ohio University in Athens and Tyler Faith at the University of Queensland in Brisbane, Australia, have discovered six more-complete skulls on an island in Lake Victoria in Kenya (one of the skulls is pictured). X-ray scans revealed large, hollow nasal passages that extended out from the top of the animals' heads. Known as nasal domes,

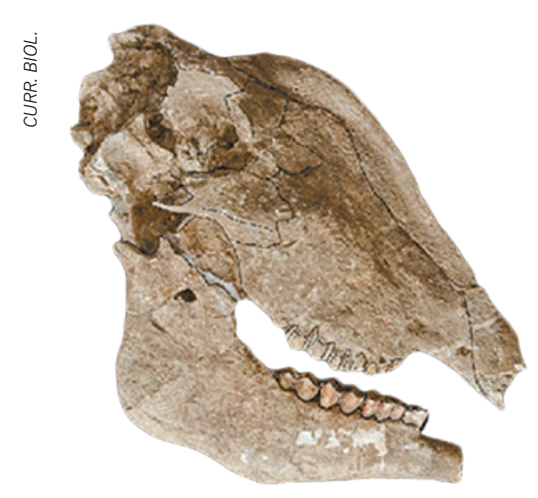

these have previously been seen only in some hadrosaurs that lived around 65 million years ago - a rare example of how similarities in behaviour and environment can spur the evolution of a common feature in distantly related animals.

Acoustic modelling suggested that the structures allowed $R$. atopocranion to vocalize at very low frequencies, possibly helping herds to communicate without alerting predators, the authors propose.

Curr. Biol. http://doi.org/bcdn (2016) \section{LINGUISTICS \\ Languages have common structure}

Many languages share a universal semantic structure that is independent of their speakers' culture, environment or how closely the languages are related.

Hyejin Youn and Tanmoy Bhattacharya at the Santa Fe Institute in New Mexico and their colleagues studied the words for 22 universal concepts - such as 'Sun' and 'water' - across 81 languages, and identified which words had multiple meanings. They linked related words and meanings together to form network maps, and found common patterns in the way that different meanings were connected to each other. For example, words for 'sea' and 'salt' were closer to each other than to words for 'Sun', and this structure was preserved across all the languages.

This method could help to reveal concepts that are universal features of human cognition and language use, the authors say.

Proc. Natl Acad. Sci. USA http://doi.org/bb94 (2016)

\section{Better battery with more juice}

Researchers have made a battery that can both hold high energy and deliver high power.

\section{SOCIAL SELECTION}

\section{'A field of crop scientists'}

When cancer researcher Ritankar Majumdar published a blog post about the collective names of doctors and scientists on 2 February (go.nature.com/fzcnqt), he had no idea he would inspire \#scientistherdnames. Hundreds of tweets with the hashtag, including "a cloud of data scientists" and "a nucleus of physicists", soon dominated scientists' Twitter streams. Some tweets reflected the angst

DNATURE.COM

For more on popular papers:

go.nature.com/8njfmx experienced in the lab. Erol Akçay, a theoretical biologist at the University of Pennsylvania in Philadelphia, tweeted: "A grant of silent sobs."
Batteries typically can hold a lot of energy but discharge it at low rates. To make a material capable of both high energy and high power, a team led by Yan Yu at the University of Science and Technology of China in Hefei placed sodium vanadium triphosphate $\left(\mathrm{Na}_{3} \mathrm{~V}_{2}\left(\mathrm{PO}_{4}\right)_{3}\right)$ inside a mixture of reduced graphene oxide and carbon nanotubes to improve the sodium compound's conductivity. They deposited the material onto a currentcollecting stainless steel support, avoiding the use of additives that would reduce the performance of the device.

The team made a battery with the porous material and showed that the device could deliver high levels of energy at a high rate. It also kept $96 \%$ of its capacity even after being charged and discharged 2,000 times. The approach could be used with other electrode materials, the authors say.

Adv. Mater. http://doi.org/f3kvx3 (2016)

\section{ENVIRONMENTAL SCIENCE}

\section{Ocean plastic hurts oysters}

Oysters that consume the small pieces of plastic littering the world's oceans produce fewer and less-robust offspring than their unexposed counterparts.

Millions of tonnes of plastic end up in the oceans every year, and tiny 'microplastic'

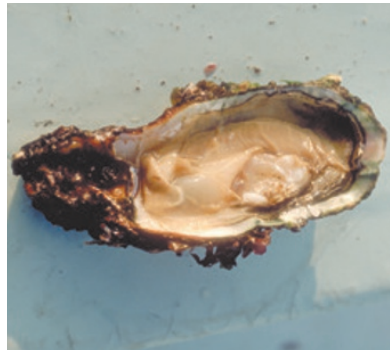

fragments - those smaller than 5 millimetres in diameter - could be harming marine life. Arnaud Huvet at the French marine-research agency IFREMER in Plouzané and his colleagues placed Pacific oysters (Crassostrea gigas; pictured) in water laced with micrometre-sized polystyrene spheres, at levels estimated to be similar to those where oysters live in the wild. After two months, oysters exposed to the plastic produced fewer and smaller egg cells, less-mobile sperm and fewer offspring than did animals raised in water without the plastic. The offspring also grew more slowly.

Ingested plastic might be disturbing the oysters' digestion and releasing endocrine-disrupting chemicals, which affect reproductive systems, the authors say.

\section{Proc. Natl Acad. Sci. USA} http://doi.org/bcdm (2016)

\section{DNATURE.COM}

For the latest research published by Naturevisit:

www.nature.com/latestresearch 